UNIL-IPT-02-1

\title{
Evidence for a connection between the $\gamma$-ray and the highest energy cosmic-ray emissions by BL Lacertae objects
}

\author{
D. S. Gorbunov ${ }^{1,4}$, P. G. Tinyakov ${ }^{1,2,5}$, I. I. Tkachev ${ }^{1,3,6}$, and S. V. Troitsky ${ }^{1,7}$
}

\begin{abstract}
A set of potentially $\gamma$-ray-loud BL Lac objects is selected by intersecting the EGRET and BL Lac catalogs. Of the resulting 14 objects, eight are found to correlate with arrival directions of ultra-high-energy cosmic rays (UHECRs), with significance of the order of $5 \sigma$. This suggests that $\gamma$-ray emission can be used as a distinctive feature of those BL Lac objects that are capable of producing UHECR.
\end{abstract}

Subject headings: cosmic rays — BL Lacertae objects: general — gamma rays: theory

The highest energy cosmic rays with energies in excess of $10^{19} \mathrm{eV}$ (ultra-high-energy cosmic rays [UHECRs]), observed by AGASA (Takeda et al. 1999) and Yakutsk (Afanasiev et al. 1996) experiments, show a significant number of clusters at angles of the order of experimental angular resolution (Uchihori et al. 2000). The significance of clustering is quantitatively estimated by calculating the angular correlation function of the UHECR events (Tinyakov \& Tkachev 2001a). It follows that the observed clustering has probability of less than $10^{-5}$ to occur as a result of a statistical fluctuation. This suggests that (1) there exist compact

\footnotetext{
${ }^{1}$ Institute for Nuclear Research of the Russian Academy of Sciences, 60th October Anniversary Prospect 7a, 117312, Moscow, Russia

${ }^{2}$ Institute of Theoretical Physics, University of Lausanne, CH-1015, Lausanne, Switzerland

${ }^{3}$ CERN Theory Division, CH-1211 Geneva 23, Switzerland

${ }^{4}$ e-mail: gorby@ms 2 .inr.ac.ru

${ }^{5}$ e-mail: Peter.Tinyakov@cern.ch

${ }^{6}$ e-mail: Igor.Tkachev@cern.ch

${ }^{7}$ e-mail: st@ms2.inr.ac.ru
} 
sources of UHECRs and (2) the already existing data may contain information sufficient to identify the actual sources, the subset of cosmic rays with maximum autocorrelations being the best choice for this purpose.

This line of reasoning was pursued by Tinyakov \& Tkachev (2001b) assuming that BL Lacertae objects are relevant candidates. Significant correlations were found with the subset of most powerful confirmed BL Lac objects. After assigning penalties for subset selection and bin size adjustment, the probability of such correlation to occur by chance in a random distribution is of order $10^{-4}$. BL Lac objects comprise a subclass of blazars characterized by the absence of emission lines. Blazars are thought to have relativistic jets directed along the line of sight, while the absence of emission lines indicates low ambient matter and radiation fields and therefore favorable conditions for the acceleration of particles to highest energies. For this reason, BL Lac objects may be particularly promising candidates for UHECR sources.

It follows from both the statistical arguments (Dubovsky et al. 2000) and correlation analysis (Tinyakov \& Tkachev 2001b,c) that only a small fraction of existing BL Lac objects should be capable of producing highest energy cosmic rays. For understanding the nature of the sources and the mechanism of UHECR emission, the key question is which physical characteristics single out the actual emitters among all BL Lac objects? In this Letter we propose that the strong $\gamma$-ray emission is the feature that distinguishes UHECR sources.

There are general reasons to expect the connection between UHECR and $\gamma$-ray emissions. Both the acceleration of particles in the source and their subsequent propagation in the intergalactic space is accompanied by energy losses. A substantial part of this energy is transferred into the electromagnetic cascade and, generically, ends up in the EGRET energy region (Berezinsky et al. 1990; Coppi \& Aharonian 1996). In models involving neutrinos via the Z-burst mechanism (Fargion et al. 1999; Weiler 1999; Fargion et al. 2001), and those based on very high-energy photons (Kalashev et al. 2001; Neronov et al. 2002), the astrophysical accelerator must be very powerful to provide sufficient flux of primary ultrahigh-energy particles. In these models, one may expect a strong electromagnetic radiation from the source and substantial contribution into EGRET flux. Note that the extragalactic cascade may get isotropized by random magnetic fields when approaching the low energy end; this may cause smearing of point sources and result in contributions into $\gamma$-ray background. In any case, these arguments suggest that $\gamma$-ray emission may be an important distinctive feature of UHECR sources ${ }^{8}$.

\footnotetext{
${ }^{8}$ We are grateful to A. Neronov and D. Semikoz for numerous useful discussions of this subject. Note that possible connection between gamma and neutrino signals has also been discussed in Fargion (2002).
} 
In order to test this hypothesis we first select those BL Lac objects that can be associated with $\gamma$-ray sources and then study their correlations with UHECR. The most complete list of the $\gamma$-ray sources can be found in the third EGRET catalog (Hartman et al. 1999) containing 271 object. Of these objects, 67 are identified with active galactic nuclei (AGNs), five with pulsars, one with a solar flare, one with the LMC, and 27 are tentatively identified with AGNs. The remaining 170 objects are unidentified.

In this Letter we do not rely on the existing EGRET identification of objects, neither do we attempt our own object-by-object analysis. Instead, we adopt a purely statistical approach: we take the full set of confirmed BL Lac objects from the Veron2001 catalog (Véron-Cetty \& Véron 2001) consisting of 350 objects, and we select a subsample of those that may be associated with an EGRET $\gamma$-ray source. The selection procedure is as follows: Point sources in the EGRET catalog are defined as a local excess of a signal over the background. Each source is associated with a contour containing $95 \%$ of the signal. For each contour, a circle of equal area is defined, with the radius $R_{95}$. These radii are listed in the EGRET catalog. They roughly correspond to uncertainties in the positions of the sources. However, the $95 \%$ contours are often noncircular. Additional systematic errors in position determination may be present in the case of a bright nearby source (such cases are marked as "confused" in the catalog). As a result, many well-identified sources (e.g., the Vela pulsar that is unambiguously identified by timing) fall outside of $R_{95}$. In our analysis, we consider an object to be associated with the EGRET source if the angular distance between the two does not exceed $2 R_{95}$. In cases of ambiguity the nearest neighbor is taken.

According to this procedure, 14 BL Lac objects from the Veron2001 catalog are associated with EGRET sources. They are listed in Table 1. Of these 14 objects, eight already have identifications in the EGRET catalog, while six are newly proposed identifications. Out of eight previously identified objects, five have the same identifications in the SIMBAD database as is suggested by our procedure (objects marked by asterisks in Table 1). Interestingly, in those three cases when our procedure suggests identification different from the existing one, the latter has a question mark in the SIMBAD database, while in five cases when they coincide the existing identification is considered firm. This rather good agreement with previous results gives confidence that at least part of previously unidentified EGRET sources listed in Table 1 should be identified with corresponding BL Lac objects.

Since the EGRET 95\% contours are large enough to contain several astrophysical objects, the identifications depend on the assumptions about candidate sources. Most previous works have concentrated on the powerful radio quasars as possible candidates (see, e.g., Mattox et al. (2001)). An approach somewhat similar to ours was used by Punsly (1999) where correlations of EGRET catalog with X-ray and moderate radio sources (ROSAT-Green Bank 
catalog) were considered. It revealed several new identifications, large fraction of them being BL Lac objects.

Being based on position coincidence only, the identifications proposed in Table 1 cannot be considered as final. Instead, Table 1 should be treated as a starting point for more detailed object-by-object study, including EGRET intensity maps, time correlations, etc. Such an analysis goes beyond the scope of this Letter. It is important to note, however, that possible misidentifications in Table 1 do not compromise our main result, strong correlation of the selected subsample with UHECRs. Like any random factor, such misidentifications can only diminish the correlations.

Let us now turn to correlations between the set of 14 (potentially) $\gamma$-ray-loud BL Lac objects of Table 1 and UHECRs. In the part concerning UHECRs, we follow the approach of Tinyakov \& Tkachev (2001b) and use the set of cosmic rays with the largest autocorrelations. This set consists of 39 AGASA events with energies $E>4.8 \times 10^{19} \mathrm{eV}$ and 26 Yakutsk events with energies $E>2.4 \times 10^{19} \mathrm{eV}$ (Tinyakov \& Tkachev 2001a).

The numerical algorithm used in this Letter is identical to that of Tinyakov \& Tkachev (2001a,b,c). We characterize the significance of correlations between UHECRs and a given set of sources at a given angular scale $\delta$ by the probability $p(\delta)$ defined in the following way. First, we count the number of source/cosmic-ray pairs separated by the angle $\leq \delta$ in the real data, thus obtaining the data count $N_{d}(\delta)$. We then generate a large number of random (mock) sets of cosmic rays, taking into account actual acceptance of the experiments in such a way that the large-scale distribution of mock cosmic rays is uniform. On small scales we introduce autocorrelations in mock sets since the real data are clustered. The number of clusters added in each mock set mimics the real data, while cluster positions are random. For each mock set, the number of source/cosmic-ray pairs is then counted in the same way as for the real data, giving the mock count $N_{m}(\delta)$. At a large total number of mock sets, the fraction of mock sets for which $N_{m}(\delta) \geq N_{d}(\delta)$ gives $p(\delta)$.

In the correlation analysis, we take into account possible effects of the Galactic magnetic field (GMF) on propagation of UHECRs. We use the spiral model of GMF with different directions of the field in the two spiral arms and consider two cases: symmetric and antisymmetric field with respect to the galactic plane. The details of the model and corresponding parameters can be found in Tinyakov \& Tkachev (2001c) together with further references. We assume that primary particles can have charges of $Q=0, \pm 1$.

In the case $Q \neq 0$, the positions of cosmic rays are corrected for the deflections in GMF prior to counting the number of pairs with given angular separation. For each cosmic ray there are several possible positions after correction for GMF corresponding to different 
allowed charges. For a given ray, the minimum angular distance over the set of sources and charges determines the resulting charge assigned to that ray. In all cases, each randomly generated set is subject to exactly the same procedure as the real data. This guarantees that no correlations are artificially introduced.

The results of the calculations for the charge assignments $Q=0, Q=1, Q=0,1$ and $Q=0, \pm 1$ and for two types of magnetic field (symmetric and antisymmetric) are presented in Table 2. The correlations are rather significant in all cases, being the best in the case of charges $Q=0,1$ and antisymmetric field, in agreement with Tinyakov \& Tkachev (2001c). In this case, the data count $N_{d}\left(2.7^{\circ}\right)=13$, while 2 is expected in average for a uniform background. The probability for this to occur by chance is $p(\delta)=3 \times 10^{-7}(5.1 \sigma)$. The dependence of $p(\delta)$ on $\delta$ in this case is shown in Fig. 1.

From the analysis of Table 2 one is tempted to conclude that the case $Q=0,1$ (neutral and positively charged particles) is favored. However, present statistics are not enough for such a conclusion, as the following simple argument shows. The data count is subject to fluctuations that may be roughly estimated as $\pm \sqrt{N_{d}}$ (these fluctuations would be observed if the AGASA and Yakutsk experiments were repeated many times). If the "average" data count were 10, counts from seven to 13 would occur equally often. Corresponding probabilities $p(\delta)$ would range from $10^{-4}$ to $10^{-7}$. Thus, unlike correlations themselves, the difference between the cases $Q=0, Q=0,1$ and $Q=0, \pm 1$ can easily be explained by a fluctuation.

Energies and charges of UHECR events that contribute into correlations with $\gamma$-rayloud BL Lac objects are listed in columns (7) and (8) of Table 1 (the antisymmetric magnetic field model is assumed). Multiple charges in column (8) mean that the corresponding event contributes to correlations under different charge assignments.

The comparison between Table 1 of this Letter and Table 1 of Tinyakov \& Tkachev (2001b) shows that the same BL Lac objects and cosmic rays contribute to correlations in Ref. (Tinyakov \& Tkachev 2001b) and in the case of $Q=0$ presented above. In Tinyakov \& Tkachev (2001b), the set of brightest BL Lac objects was selected by imposing cuts on redshift, apparent magnitude, and radio flux. In the resulting subset of 22 BL Lac objects, five candidate sources were identified. It is remarkable that four out of these five candidates, in particular all three that correlate with UHECR multiplets, are among the 14 BL Lac objects that comprise the intersection of BL Lac and EGRET catalogs, $\gamma$-ray-loud BL Lac objects. Even more remarkable is that out of 10 remaining BL Lac objects, four correlate with cosmic rays after correction for GMF. Among the remaining six that do not correlate with UHECRs, two objects are situated in the Southern hemisphere invisible for Yakutsk and AGASA experiments. These objects can be excluded from correlation analysis. Thus, the majority of $\gamma$-ray-loud BL Lac objects (eight out of 12) correlate with UHECR. One 
concludes that the ability to emit $\gamma$-rays may be used as the physical criterion that allows to select actual UHECR sources from the set of all BL Lac objects.

BL Lac objects are typically faint objects. Some of the unidentified EGRET sources may be actually BL Lac objects that have not not yet been observed at other wavelengths, or have been observed but not identified as BL Lac objects. If this is the case and our conclusion about the connection between $\gamma$-ray and UHECR emissions is correct, one may expect correlations between unidentified EGRET sources and UHECRs. To check this, we calculated correlations between UHECRs and unidentified EGRET sources having Galactic latitude $|b|>10^{\circ}$ (the cut $|b|>10^{\circ}$ is made to increase the fraction of extragalactic sources as, according to Grenier (2000), the total number of such sources is expected to be 30-40 only). This set contains 96 objects. Correlations are best when all particles are assumed to have a charge of $Q=+1$; corresponding significance is $p(\delta)=10^{-4}$ at $\delta=3^{\circ}$. Table 3 summarizes EGRET sources and cosmic rays that contribute to correlations. It is interesting to note that, unlike Table 1, Table 3 seems to favor positively charged particles. We expect that some EGRET sources listed in Table 3 are BL Lac objects that have not yet been observed.

To summarize, there exists a significant correlation of arrival directions of UHECRs with $\gamma$-ray-loud BL Lac objects (BL Lac objects that may be associated with the EGRET sources). This confirms the conjecture that strong $\gamma$-ray emission is a characteristic feature of those BL Lac objects that are the sources of UHECR. Present data are compatible with charges of primary particles $Q=0, Q=+1, Q=0,+1$ and $Q=0, \pm 1$, although they favor the latter two cases. It does not seem possible, with the present statistics, to distinguish between these cases on the basis of correlation analysis, but it should be possible in the future. This question is of particular interest since specific charge composition is a good signature of most of the existing models. Charge $Q=0, \pm 1$ would speak strongly for neutrino models. Charge $Q=1$ would favor protons accelerated in BL Lac objects (note that energies of most of the $Q=1$ events in Table 1 would allow them to reach us from super-GZK distances provided extragalactic magnetic fields are small). The cases $Q=0,1$ and $Q=0$ would suggest, in view of the distance to BL Lac objects and presence of neutral particles, the existence of new physics (e.g., exotic neutral particles (Chung et al. 1998; Berezinsky et al. 2002; Gorbunov et al. 2001) or violation of Lorentz invariance (Coleman \& Glashow 1999; Dubovsky \& Tinyakov 2002)).

The results presented here suggest that the sources of UHECRs are high-energy-peaked BL Lac objects located opposite the flat-spectrum radio quasar end of the "unified blazar sequence" (Ghisellini et al. 1998). This does not contradict the conclusions of Sigl et al. (2001), who found no correlations between UHECR and identified EGRET blazars. Indeed, 
most of the latter are high-polarization blazars, and not low-polarization, high-energypeaked BL Lac objects that, according to our study, are the most probable sources of UHECR.

The work of P.T. is supported by the Swiss Science Foundation, grant 21-58947.99. The work of D.G. and S.T. is supported in part by the program SCOPES of the Swiss National Science Foundation, project No. 7SUPJ062239, by SSLSS grant 00-15-96626, and by RFBR grant 02-02-17398. The work of D.G., I.T., and S.T. is supported in part by INTAS grants YSF 2001/2-142, 99-1065 and YSF 2001/2-129, respectively. This Letter made use of the SIMBAD database, operated at the Centre de Données Astronomiques de Strasbourg, Strasbourg, France.

\section{REFERENCES}

Afanasiev, B. N., et al. 1996, in Proc. Int. Symp. on Extremely High Energy Cosmic Rays: Astrophysics and Future Observatories, Ed. by Nagano, p.32

Berezinsky, V. S., Bulanov S. V., Dogiel, V. A., Ginzburg, V. L., \& Ptuskin, V. S. 1990, Astrophysics of Cosmic Rays (Amsterdam: Elsevier)

Berezinsky, V., Kachelriess, M., \& Ostapchenko, S. 2002, Phys. Rev. D, 65, 083004

Chung, D. J., Farrar, G. R., \& Kolb, E. W. 1998 Phys. Rev. D, 57, 4606

Coleman, S. R. \& Glashow, S. L. 1999, Phys. Rev. D, 59, 116008

Coppi, P. S. \& Aharonian, F. A. 1996, ApJ, 487, L9

Dubovsky, S. L., Tinyakov, P. G., \& Tkachev, I. I. 2000, Phys. Rev. Lett., 85, 1154

Dubovsky, S. L. \& Tinyakov, P. G. 2002, Astropart. Phys. 18, 89

Fargion, D., Mele, B., \& Salis, A. 1999, ApJ, 517, 725

Fargion, D., Grossi, M., De Sanctis Lucentini, P. G., Di Troia, C. 2001, Suppl. B, J. Phys. Soc. Jpn., 70, 46

Fargion, D. 2002, ApJ, 570, 909

Fossati, G., Maraschi, L., Celotti, A., Comastri, A., \& Ghisellini, G. 1998, MNRAS, 299, 433

Gorbunov, D. S., Raffelt G. G. \& Semikoz, D. V. 2001, Phys. Rev. D, 64, 096005 
Grenier, I. A. 2000, A\&A, 364, L93

Ghisellini, E., Celotti, A., Fossati, G., Maraschi, L., \& Comastri, A. 1998, MNRAS, 301, 451

Hartman, R. C. et al. 1999, ApJS, 123, 79

Kalashev, O. E., Kuzmin, V. A., Semikoz, D. V., \& Tkachev, I. I. 2001, astro-ph/0107130

Mattox, J. R., Hartman, R. C., \& Reimer, O. 2001, ApJS, 135, 155

Neronov, A., Semikoz, D., Aharonian F., and Kalashev, O. 2002, Phys. Rev. Lett., 89, 051101

Punsly, B. 1999, ApJ, 516, 141

Sigl, G., Torres, D. F., Anchordoqui, L. A., \& Romero, G. E. 2001, Phys. Rev. D, 63, 081302

Takeda, M. et al. 1999, ApJ, 522, 225

Tinyakov, P. G., \& Tkachev, I. I. 2001a, JETP Lett., 74, 1 (translation from Pisma Zh. Eksp. Teor. Fiz., 74, 3)

Tinyakov, P. G., \& Tkachev, I. I. 2001b, JETP Lett., 74, 445 (translation from Pisma Zh. Eksp. Teor. Fiz., 74, 499)

Tinyakov, P. G., \& Tkachev, I. I. 2001c, astro-ph/0111305.

Uchihori, Y., Nagano, M., Takeda, M., Teshima, M., Lloyd-Evans, J., \& Watson, A. A. 2000, Astropart. Phys., 13, 151

Véron-Cetty, M.-P., \& Véron, P. 2001, A\&A, 374, 92

Weiler, T. 1999, Astropart. Phys. 11, 30 


\begin{tabular}{|c|c|c|c|c|c|c|c|}
\hline $\begin{array}{l}3 \mathrm{EG} \mathrm{J} \\
(1)\end{array}$ & $\begin{array}{c}\text { E ID } \\
(2)\end{array}$ & $\begin{array}{c}\text { Possible BLL } \\
\text { (3) }\end{array}$ & $\begin{array}{l}1 \\
(4)\end{array}$ & $\begin{array}{c}\mathrm{b} \\
(5)\end{array}$ & $\begin{array}{l}\mathrm{z} \\
(6)\end{array}$ & $\begin{array}{c}\mathrm{E} \\
(7)\end{array}$ & $\begin{array}{l}Q \\
(8)\end{array}$ \\
\hline $0433+2908$ & $\mathrm{AGN}$ & $2 \mathrm{EG}$ J0432+2910* & 170.5 & -12.6 & - & $\begin{array}{l}5.47 \\
4.89\end{array}$ & $\begin{array}{l}0, \pm 1 \\
0,+1\end{array}$ \\
\hline $0808+5114$ & AGN? & 1ES $0806+524^{*}$ & 166.2 & 32.91 & 0.138 & $\begin{array}{l}3.4 \\
2.8 \\
2.5\end{array}$ & $\begin{array}{l}0 \\
0 \\
0\end{array}$ \\
\hline 0812-0646 & AGN? & 1WGA J0816.0-0736 & 229.8 & 14.96 & 0.04 & - & \\
\hline $1009+4855$ & AGN? & GB $1011+496$ & 165.5 & 52.71 & 0.2 & - & \\
\hline $1052+5718$ & AGN? & RGB J1058+564* & 149.6 & 54.42 & 0.144 & $\begin{array}{l}7.76 \\
5.35 \\
5.50\end{array}$ & $\begin{array}{l}0,-1 \\
0,-1 \\
-1\end{array}$ \\
\hline $\begin{array}{l}1222+2841 \\
1310-0517\end{array}$ & AGN & $\begin{array}{c}\text { ON 231* } \\
\text { 1WGA J1311.3-0521 }\end{array}$ & $\begin{array}{l}201.7 \\
312.1\end{array}$ & $\begin{array}{l}83.29 \\
57.16\end{array}$ & $\begin{array}{c}0.102 \\
0.16\end{array}$ & - & \\
\hline $1424+3734$ & & TEX $1428+370$ & 63.95 & 66.92 & 0.564 & 4.97 & $0,+1$ \\
\hline $1605+1553$ & AGN & PKS $1604+159^{*}$ & 29.38 & 43.41 & - & - & \\
\hline $1621+8203$ & & 1ES $1544+820$ & 116.5 & 32.97 & - & 2.7 & +1 \\
\hline $1733+6017$ & & RGB J1742+597 & 88.46 & 31.78 & - & $\begin{array}{c}2.5 \\
6.93\end{array}$ & $\begin{array}{l}+1 \\
-1\end{array}$ \\
\hline $1850+5903$ & & RGB J1841+591 & 88.68 & 24.29 & 0.53 & $\begin{array}{l}5.8 \\
2.8\end{array}$ & $\begin{array}{l}+1 \\
+1\end{array}$ \\
\hline $1959+6342$ & & 1ES $1959+650$ & 98.0 & 17.67 & 0.047 & 5.5 & +1 \\
\hline $2352+3752$ & AGN? & TEX $2348+360$ & 109.5 & -24.91 & 0.317 & - & \\
\hline
\end{tabular}

Table 1: List of BL Lac objects associated with EGRET sources and UHECR which contribute to correlations.

Note: (1) EGRET name; (2) EGRET identification; (3) suggested BL Lac counterpart; the five objects marked with as asterisk are the cases when suggested identification argrees with the SIMBAD database; (4) and (5) Galactic coordinates of the BL Lac counterpart; (6) redshift of the BL Lac counterpart as given by Véron-Cetty \& Véron (2001); (7) energies of correlating cosmic rays in units of $10^{19} \mathrm{eV}$; (8) UHECR charge assignments under which the correlation occurs. 


\begin{tabular}{c|c|c|c|c|c|c}
$\mathrm{Q}$ & \multicolumn{2}{|c|}{ antisymmetric field } & \multicolumn{3}{|c}{ symmetric field } \\
\hline & $p(\delta)$ & $N_{d}(\delta)$ & $\delta$ & $p(\delta)$ & $N_{d}(\delta)$ & $\delta$ \\
\hline 0 & $10^{-4}$ & 8 & $2.9^{\circ}$ & $10^{-4}$ & 8 & $2.9^{\circ}$ \\
+ & $7 \cdot 10^{-5}$ & 8 & $2.7^{\circ}$ & $9 \cdot 10^{-4}$ & 9 & $3.7^{\circ}$ \\
$0,+$ & $3 \cdot 10^{-7}$ & 13 & $2.7^{\circ}$ & $2 \cdot 10^{-6}$ & 12 & $2.6^{\circ}$ \\
$0, \pm$ & $10^{-6}$ & 15 & $2.8^{\circ}$ & $2 \cdot 10^{-6}$ & 15 & $2.9^{\circ}$
\end{tabular}

Table 2: Summary of correlations between 14 BL Lac objects and 65 cosmic rays for different charge assignments and models of the GMF.

\begin{tabular}{l|c|c|c|l}
$3 \mathrm{EG} \mathrm{J}$ & $\mathrm{l}$ & $\mathrm{b}$ & $\mathrm{E}$ & $\mathrm{Q}$ \\
\hline $0245+1758$ & 157.6 & -37.11 & 3.2 & +1 \\
$0329+2149$ & 165.0 & -27.88 & 4.8 & +1 \\
$0429+0337$ & 191.4 & -29.08 & 6.19 & $0,+1$ \\
$1227+4302$ & 138.6 & 73.33 & 4.3 & +1 \\
$1308+8744$ & 122.7 & 29.38 & 3 & +1 \\
$1337+5029$ & 105.4 & 65.04 & 5.68 & +1 \\
$1621+8203$ & 115.53 & 31.77 & 2.7 & +1 \\
$1824+3441$ & 62.49 & 20.14 & 9.79 & $0, \pm 1$ \\
$1835+5918$ & 88.74 & 25.07 & 5.8 & +1 \\
& & & 2.8 & +1
\end{tabular}

Table 3: List of unidentified EGRET sources correlating with cosmic rays. Note: Columns are the same as in Table 1. 


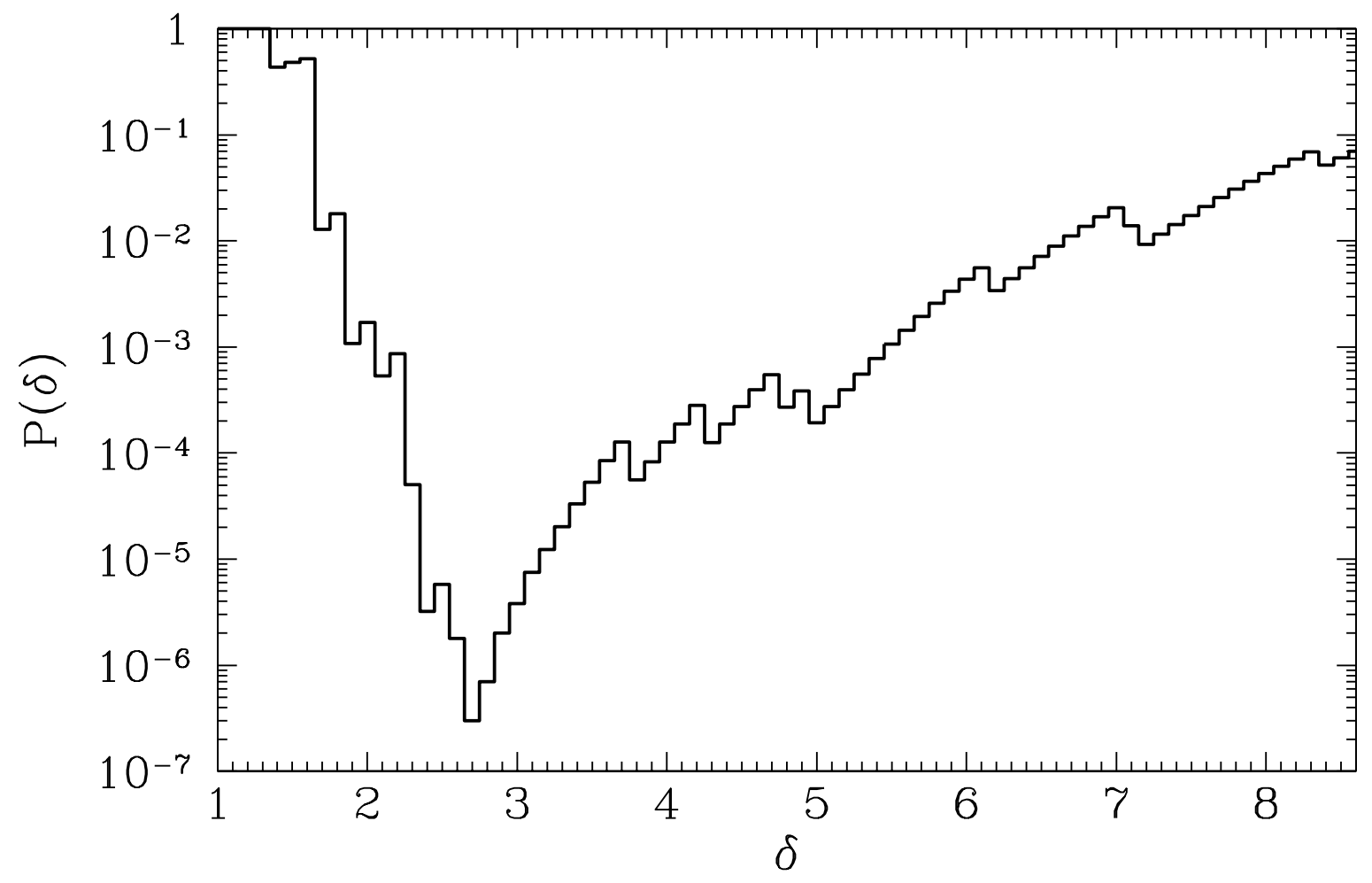

Fig. 1.- Significance of correlations between $14 \gamma$-ray-loud BL Lac objects and UHECRs as a function of the angular scale $\delta$ for the $Q=0,1$ charge composition. This corresponds to the lowest probability entry of Table 2. 